\title{
Multiscale perspectives of virus entry via endocytosis
}

Eric Barrow ${ }^{1}$, Anthony V Nicola $2,3^{*}$ and Jin Liu ${ }^{1 *}$

\begin{abstract}
Most viruses take advantage of endocytic pathways to gain entry into host cells and initiate infections. Understanding of virus entry via endocytosis is critically important for the design of antiviral strategies. Virus entry via endocytosis is a complex process involving hundreds of cellular proteins. The entire process is dictated by events occurring at multiple time and length scales. In this review, we discuss and evaluate the available means to investigate virus endocytic entry, from both experimental and theoretical/numerical modeling fronts, and highlight the importance of multiscale features. The complexity of the process requires investigations at a systems biology level, which involves the combination of different experimental approaches, the collaboration of experimentalists and theorists across different disciplines, and the development of novel multiscale models.
\end{abstract}

Keywords: Viral entry, Theoretical modeling, Endocytosis, Multiple scales, Virus trafficking, Multiscale modeling

\section{Introduction}

Viruses are small but masterful infectious agents infecting all types of organisms from bacteria, plants and animals to humans. Emerging and re-emerging infectious diseases caused by viruses have taken a tremendous toll on human health worldwide [1-3]. For instance, human immunodeficiency virus (HIV) has killed more than 25 million people since 1981. 3\% of the world's population has been infected with hepatitis $\mathrm{C}$ virus, which causes 350,000 deaths annually. Influenza A virus infection results in hundreds of thousands of deaths each year and has caused some of the deadliest pandemics in human history.

Viruses are obligatory intracellular parasites and therefore must enter host cells and deliver their genetic material to initiate infection. The plasma membrane of a host cell represents the first physical barrier that viruses must overcome to gain entry. Some enveloped viruses such as herpes simplex virus type 1 (HSV-1), Sendai virus and HIV are able to penetrate into cells by direct fusion with the plasma membrane. However, the majority of viruses take endocytic pathways to enter host cells

\footnotetext{
*Correspondence: nicola@vetmed.wsu.edu; jin.liu2@wsu.edu

${ }^{3}$ Paul G. Allen School for Global Animal Health, Washington State University, Pullman, WA 99164, USA

${ }^{1}$ School of Mechanical and Materials Engineering, Washington State University, Pullman, WA 99164, USA

Full list of author information is available at the end of the article
}

for productive infection. For recent reviews see [4-8]. Endocytosis is the process by which an intracellular vesicle is formed by membrane invagination resulting in engulfment of extracellular and membrane-bound components $[9,10]$. Endocytosis plays a central role in control of essential cellular functions, modulation of membrane composition and regulation of many intracellular signaling cascades. Since endocytosis can transport relatively large particles, it is therefore critically important for the design of nanomedicines and targeted drug delivery [11]. Viruses entering cells via endocytosis offers many advantages [8]. For instance, endocytosis allows animal viruses to bypass obstacles posed by plasma membrane and cytoplasmic crowding, and by the meshwork of microfilaments in the actin cortex. It can also allow direct transport to subcellular sites of viral replication. Furthermore, endocytosed viruses can overcome host immune surveillance by leaving minimal evidence on the cell surface. Indeed, it has been suggested that HIV entry by endocytosis is favored over direct fusion with the plasma membrane [12].

Viruses take advantage of a number of endocytic mechanisms for entry. In clathrin-mediated endocytosis (CME), clathrin-coated vesicles (CCVs) are formed by the nucleation of clathrin-coated pits (CCPs) induced by receptors and bound ligands [10]. CME is the main pathway for internalization of extracellular components; 
therefore it is the most well characterized and extensively studied endocytic pathway. Macropinocytosis is a transient, growth factor induced and actin-dependent process, which often involves the formation of membrane ruffling and large vacuoles [13]. This mechanism is usually used for internalization of fluid and membrane, but it can also drive the uptake of viruses [14-16]. Several nonenveloped viruses, such as simian virus 40 (SV40) [17] and polyoma virus [18], enter host cells through caveolin-mediated endocytosis, in which caveolae composed of a coat of caveolin proteins are formed [19]. Other novel endocytic pathways, which are clathrin and caveolin independent, have also been identified [20-22] but remain poorly characterized due to the relative lack of known marker proteins. Depending on the virus, cell type, and local microenvironment variables (such as local $\mathrm{pH}$ ), viruses are able to exploit a number of different endocytic pathways to gain entry.

Despite much progress, a systematic and mechanistic understanding of viral entry into host cells through endocytosis is still elusive. As illustrated in Figure 1 in terms of CME, this is a complex process, which involves viral ligands and likely hundreds of cellular proteins, such as virus receptors, and the multifactorial machineries and signaling pathways associated with the clathrin, actin, dynamin, caveolin, and microtubule networks to name a few [23]. The entire process is dictated by the collective and cooperative interplay of various events, such as virus motions, membrane deformations, receptor diffusion and ligand-receptor interactions, occurring at multiple length and time scales. Due to the multiscale nature of the problem, it is extremely difficult to explore the whole process using a single technique. Viral endocytosis and related topics have been previously reviewed and discussed $[6,8,13,24-27]$ with focuses on different aspects. Here, we review recent technical advances in viral endocytic entry with an emphasis on the multiscale features of the process, and the collaborative and complementary roles played by experimentation and theoretical/ numerical modeling. Section 2 describes the different experimental techniques utilized in different scales to explore viral endocytic entry. In section 3, we review progress on the modeling and simulation fronts. Conclusions and future perspectives are given in section 4 .

\section{Experimental techniques}

Exploring viral endocytic entry mechanisms is challenging because of the multiple, diverse entry pathways and the multiple stages therein. Each pathway involves numerous physical and chemical interactions between the virus and host cells. Recent advances in experimental techniques at different scales have improved our understanding of this important process.

\section{Cellular scale information: electron microscopy and fluorescence microscopy}

Electron microscopy (EM), including scanning electron microscopy (SEM) and transmission electron microscopy (TEM), can provide still images with resolution close to the nanometer scale. For more than 50 years, EM has been

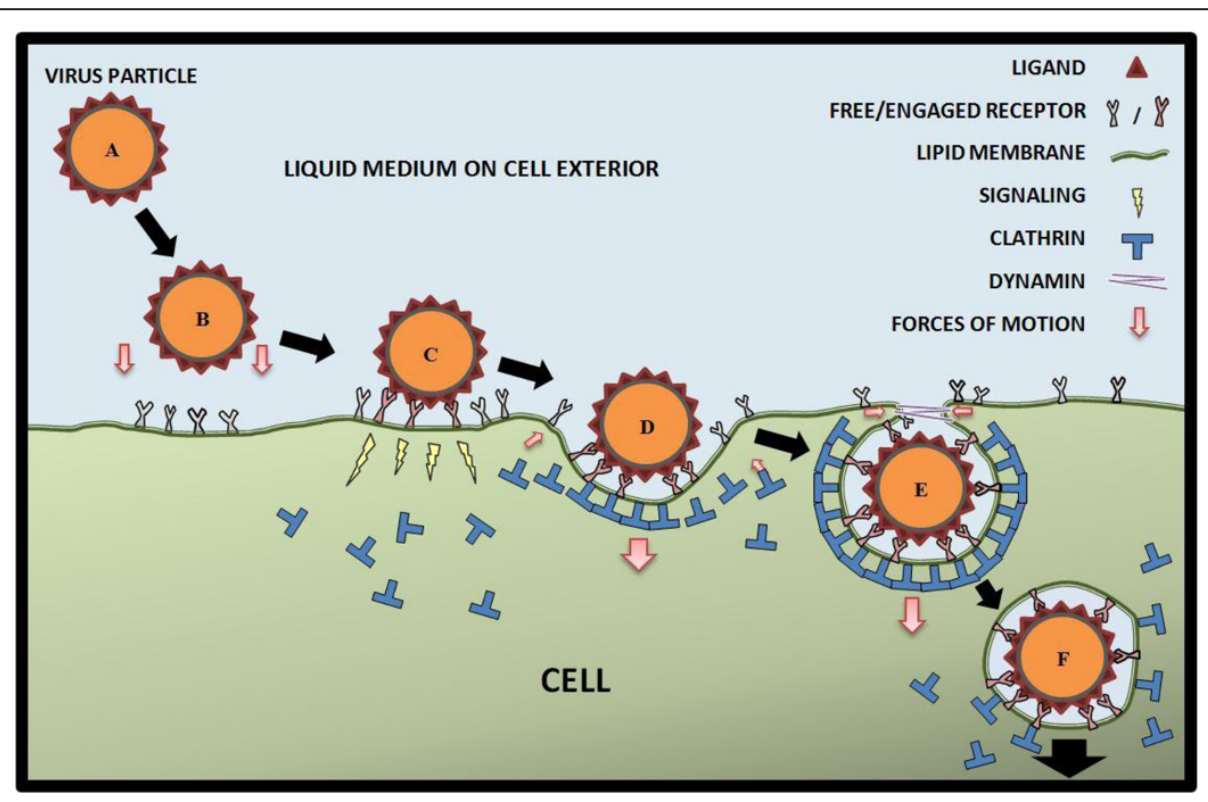

Figure 1 Illustration of the steps of virus entry via clathrin-mediated endocytosis. (A) Virus approaches the cell surface. (B) Biochemical interactions between ligands and receptors attract virus to the cell surface. (C) Virus attaches to the cell surface and signals the cell. (D) A clathrincoated pit is formed around the bound virus. (E) A clathrin-coated vesicle is formed, and the dynamin at the neck region facilitate vesicle scission. (F) The vesicle travels to cell interior. 
routinely exploited in research on virus entry to provide structural and compositional information [28]. As a more recent example, Figure 2 shows electron micrography of HSV entry via either endocytosis or by direct penetration at the plasma membrane depending on the cell type [29]. When combined with functional studies that measure successful entry of particles that initiate productive infection, EM analysis has supported the notion that both endocytic and non-endocytic pathways are productive for HSV entry.

However, EM experiments are usually performed under restricted conditions and on fixed cells, which is often incompatible with a live-cell environment. Furthermore, important dynamic interactions are usually overlooked in EM measurements. On the other hand, live-cell microscopy experiments are able to provide more dynamic information. Fluorescent-labeling of viruses and cellular structures combined with fluorescence microscopy has allowed for tracking of single virus in live cells and direct visualization and quantitative analysis of cellular dynamics [30]. Single viral particletracking revealed the caveolar endocytic entry of SV40 [31]. By tracking the dynamics of single influenza viruses and their interactions with cellular endocytic structures, Rust et al. [22] showed that influenza can enter cells using both clathrin-mediated and clathrin/caveolin- independent endocytic pathways with comparable efficiency. In addition, a virus-induced CCP formation mechanism was suggested by tracking the interaction between viruses and CCPs in real time. Single particle analysis combined with the use of a dominant-negative mutant identified epsin 1, a clathrin-interacting protein, as a cargo-specific adaptor during the endocytic entry of influenza virus [32]. Using a similar combination of methodologies, Dengue virus was found to enter live cells exclusively through CME [33]. Single particle tracking demonstrated distinct motions of murine polyoma virus-like particles [34] and human papillomavirus 16 [35] on the cell surface. Moreover, it was also found that poliovirus entered the cell by a clathrin, caveolin, flotillin and microtubule-independent, but tyrosine kinase and actin-dependent endocytic pathway [36,37].

Although single-virus analysis has revealed previously undetectable events in viral entry, the spatial resolution of conventional optical microscopy remains limited to a few hundred nanometers by the diffraction effect of light. However, virus-cell molecular interactions occur at nanometer scales. Several promising super-resolution optical microscopy techniques have been developed [38-41] and these techniques have pushed the spatial resolution to nanometer scales. Most recently one of the
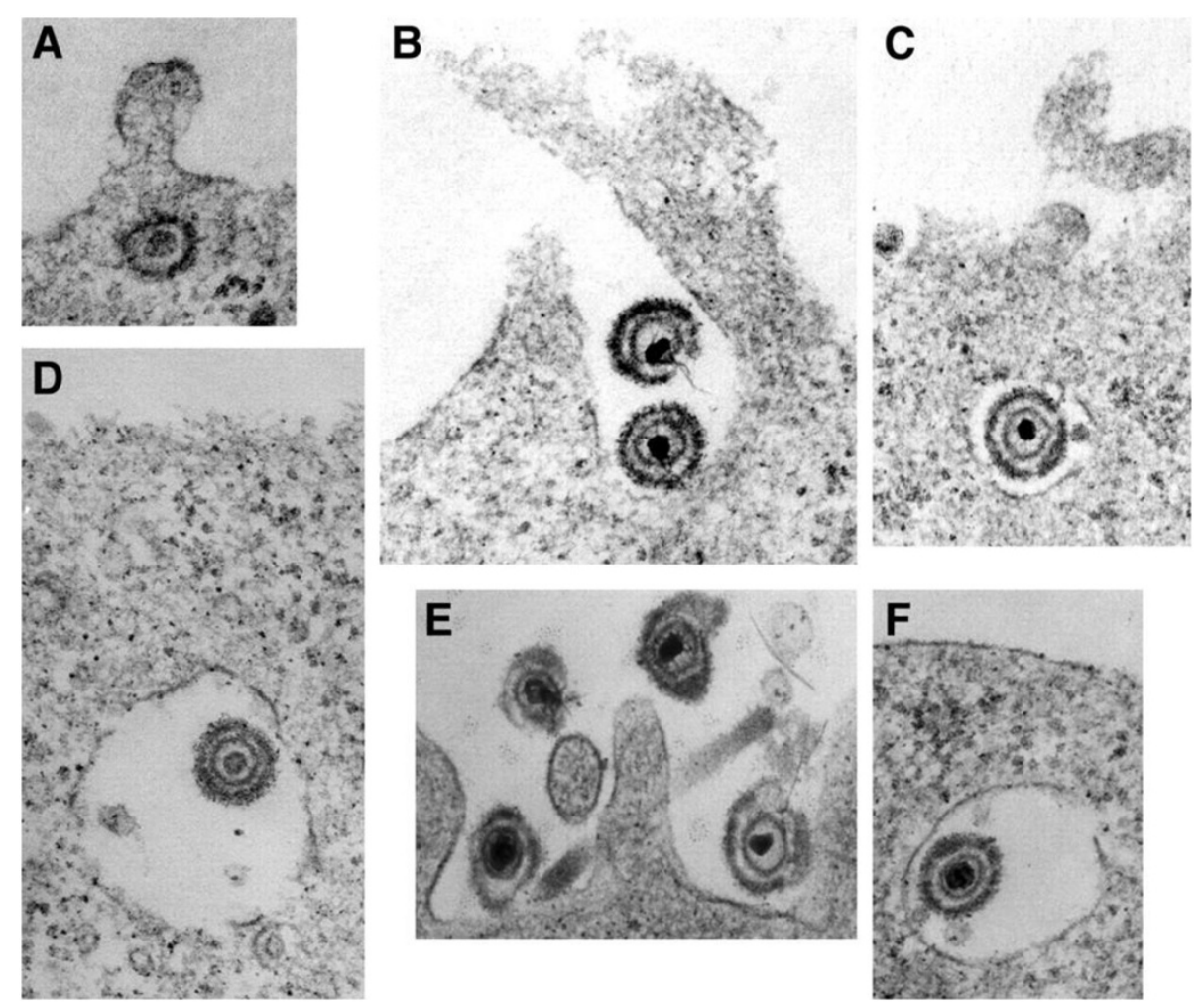

Figure 2 Electron microscopic analysis of HSV-1 uptake into cells. HSV-1 was bound to Vero (A), CHO-nectin-1 (B to D), or HeLa (E and F) cells for $2 \mathrm{~h}$ at $4^{\circ} \mathrm{C}$. This was followed by a shift to $37^{\circ} \mathrm{C}$ for $20 \mathrm{~min}$, and then the cells were processed for electron microscopy. Reproduced with permission from reference [29]. 
techniques, stochastic optical reconstruction microscopy (STORM), has been successfully utilized to quantify the size of the HIV-1 matrix shell and capsid core with a lateral resolution of $15-20 \mathrm{~nm}$ [42]. The results agreed with HIV-1 organization seen by EM.

\section{Electron tomography}

Besides physical and biochemical interactions, the geometrical parameters (such as the shape and size) of the viral particles, as well as the structural and distributional information of the envelope glycoprotein complexes also play vital roles in virus endocytic entry. For example, influenza A has pleiomorphic virions. Spherical virions utilize CME to enter host cells; however, filamentous virions use macropinocytosis as the primary entry mechanism [43].

Electron tomography (ET), an extension of traditional $E M$, is a technique to visualize and characterize the three-dimensional (3D) structures of subcellular macromolecular objects by reconstruction of a series of projected images taken at different angles of the target. ET is particularly powerful and advantageous in obtaining 3D geometrical and distributional information for non-symmetric heterogeneous objects in native form with resolution in the nano and subnanometer scales (atomic scales).

The architectures of various viral particles, including the shapes of the virions and the envelope glycoprotein complexes, have been actively explored since the emergence of the ET technique [44]. For example, by visualizing isolated HSV by cryo-ET, a combination of cryo-microscopy and electron tomography, the glycoproteins on the HSV envelope were revealed to be distributed in a nonrandom fashion, varying in length, spacing and angles [45]. In reference [46], an iterative 3D averaging algorithm was developed and utilized with cryoET measurements to determine the structure of the envelope glycoprotein complex of Moloney murine leukemia virus (MoMuLV) with a resolution of $2.7 \mathrm{~nm}$. Other types of viruses, including vaccinia virus [47], human and simian immunodeficiency viruses (HIV and SIV) [48,49], influenza virus [50] and Ebola virus [51], have also been investigated using cryo-ET techniques.

The architectures, including the interacting ligandreceptor pairs and deforming cellular membrane, surrounding the contact zone between the virus and host cell during entry have significant implications in understanding mechanisms of virus endocytic entry. ET provides an ideal tool to visualize and analyze virus-cell interactions. Recently, ET has been employed to study different stages of the virus life circle [52]. Figure $3(\mathrm{E})$ shows a tomographical image of HIV-1 in contact with $\mathrm{T}$ cells [53] which is reconstructed from EM images (A-D). As shown, a neck-shaped contact zone, which is named an "entry claw", is identified. This contact zone is $\sim 40 \mathrm{~nm}$ wide and is composed of a number of closely spaced rod-shaped structures. However, when anti-CD4 antibodies, the CCR5 antagonist TAK779, or the peptide entry inhibitor $\mathrm{C} 34$ are introduced, the contact zone cannot be observed. By analyzing a series of ET images at different stages of HSV-1 [54] or gammaherpesvirus [55] infection, molecular details of the structural changes including membrane deformation are revealed during the entry processes. Similarly, the interactions between vaccinia virus [56], filovirus [57] and their respective host cells have been studied using ET techniques.

\section{Molecular scale interactions: single-molecule force spectroscopy}

Virus entry pathways are primarily determined by the interactions between virus particles and the corresponding cellular receptors [58]. The interactions between viral ligands and cellular receptors are specific and dynamic,
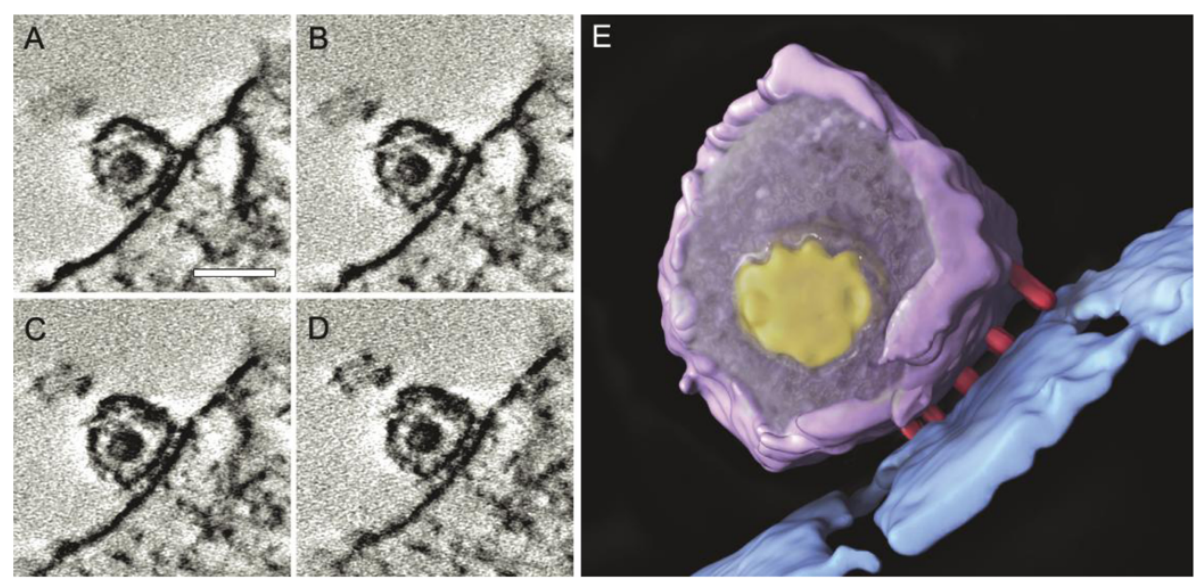

Figure 3 Imaging of HIV-1 in contact with T cells. (A-D) Four slices at different depths in a tomogram of the contact between HIV and T cells. (E) 3-D tomographically derived architecture of the contact region. Scale bar in (A) is $100 \mathrm{~nm}$. Reproduced with permission from reference [53]. 
and involve molecular scale conformational changes and signaling events. Experimental measurement of the interactions at a single molecule scale is challenging but vital to understanding viral endocytic entry.

One method of quantifying ligand-receptor interactions is the optical biosensor assay, in which the kinetic rate constants are determined by analysis of surface plasmon resonance of a glass surface before and after ligand binding. Due to its ease of use and the high throughput nature of the experimental setup, this method has been widely used to characterize the specific interactions between viral ligands and cellular receptors. For instance, in references $[59,60]$ the kinetic rate constants $\left(k_{o n}\right.$ and $k_{\text {off }}$ ) for HSV glycoprotein D binding to its cellular receptors are measured using optical biosensor assays. However, due to the inherent limitations of the controlled environment, certain dynamic information about virus entry (e.g., conformational changes and signaling events) cannot be captured by this technique.

On the other hand, atomic force microscopy (AFM) provides a powerful and versatile tool to study molecular scale interactions. In AFM, a very tiny tip mounted at the end of a microcantilever is controlled to probe a sample. The deflection of the cantilever is recorded and translated to topographical or mechanical information. Recently, AFM has received much attention in the field of biophysics [61]. In bio-imaging applications, AFM is implemented to investigate the distribution and interaction of lipids and proteins on lipid bilayers at the single molecule level [62]. In bio-force measurement applications, the elastic properties of the lipid bilayer, the bending modulus, the lateral tension and the adhesion constant, can be simultaneously determined by using AFM force measurement on a lipid membrane surface [63]. AFM is also applied to probe the differences in mechanical properties between normal and cancer cells $[64,65]$. Physical and mechanical insights from AFM measurements may be important in understanding cancer metastasis [66].

The initial interaction of HIV with the host cell is the binding of the viral surface ligand gp120 to cell surface CD4 molecules $[67,68]$. Wirtz and co-workers have implemented AFM to analyze the interactions between HIV-1 envelope glycoprotein and corresponding receptors [69]. In their experiments, the glycoprotein gp120 is attached to the cantilever tip. The tip is controlled to probe a living cell containing the receptor CD4 and/or the coreceptor CCR5. Figure 4 (left) illustrates the forcedistance curves recorded by AFM measurements of interactions between gp120 and CD4 under different conditions. As shown, a rupture event indicating binding interactions can be observed under normal conditions. However, no binding can be detected if the CD4 is blocked by an anti-CD4 function-blocking monoclonal antibody (mAb) (right-top), the gp120 is absent (rightmiddle), or the gp120 is blocked by sCD4. Furthermore, by measuring the binding strength of gp120-CD4 with CCR5 and comparing with gp120-CD4 without CCR5, the dynamics and mechanisms of the receptor/coreceptor mediated interactions, and their implications on HIV entry are discussed. In later work [70], the detailed tensile strength and lifetime of the gp120-CD4 bond are

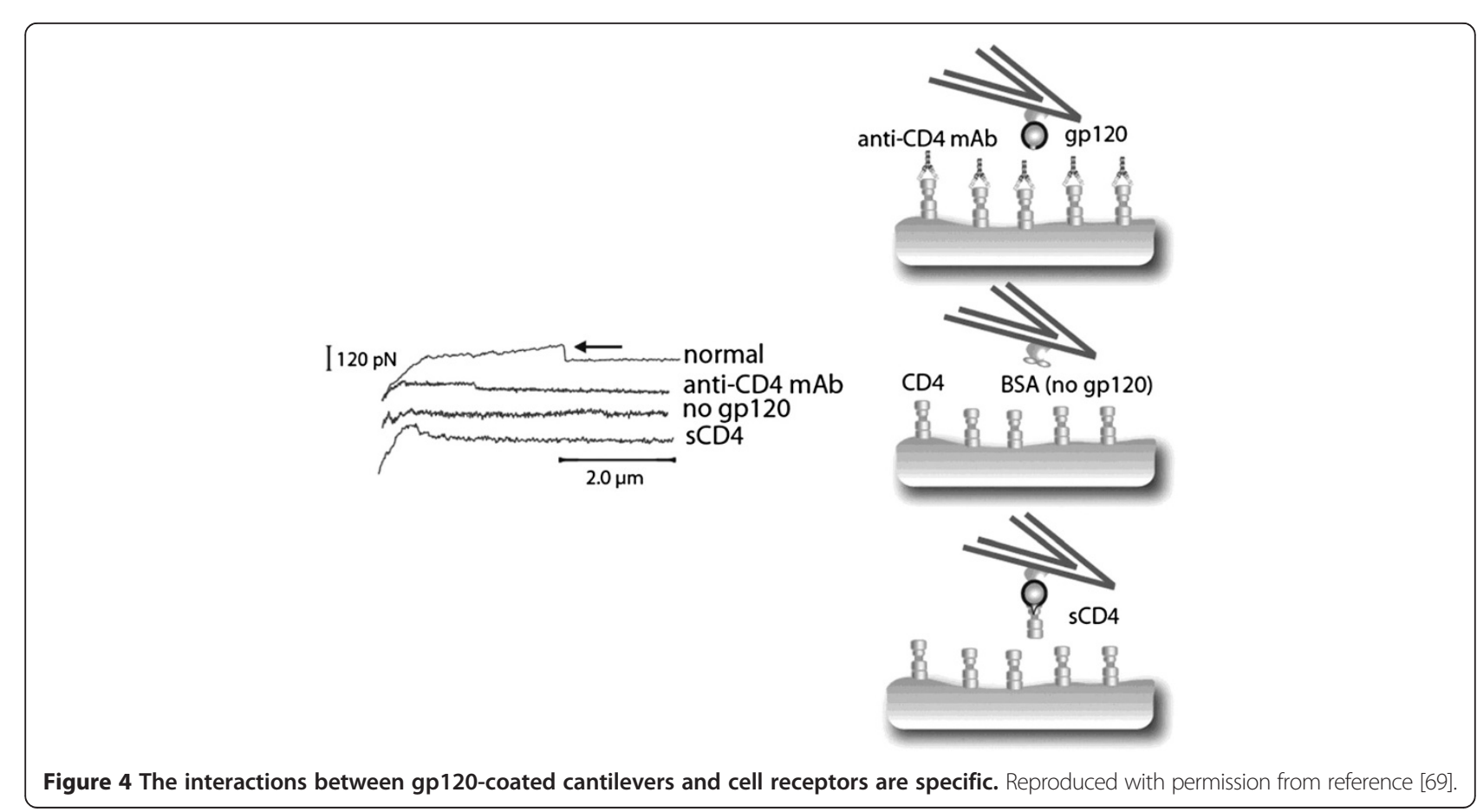


measured. A destabilization of the bond is enhanced by the coreceptor CCR5, and this is directly related to a conformational change in the gp120-CD4 bond.

\section{Theoretical and numerical models}

The entire process of viral endocytic entry is dictated by dynamic interplay among various biomechanical and biochemical interactions occurring in multiple spatial and time scales. Significant advances in experimental techniques as mentioned above have provided indispensable data from different scales. Meanwhile, a number of theoretical and numerical models have been developed recently to further improve our fundamental understanding of the mechanisms of viral endocytic entry.

\section{Theoretical continuum models}

Theoretical models have been developed to provide fundamental insights into viral endocytic entry into host cells. In such models, the total free energy functional of the system is formulated by considering both energetic and entropic contributions in endocytic events, such as receptor diffusions, ligand-receptor interactions and membrane deformations. Those energetic and entropic contributions are approximated by continuum functions. Then by considering the thermodynamic equilibrium or by minimizing the free energy functional, the effects of different parameters on viral endocytic entry can be explored.

Using a theoretical continuum model, van Effenterre and Roux [71] have discussed the effect of the volume concentration on viruses binding to a planar membrane. A relationship between the virus resting time on the cell surface and virus volume concentration was derived, based on which an optimal volume concentration for virus internalization was identified. Gao et al. [72] developed a model in the context of explaining the mechanism of clathrin-free viral endocytic entry into cells. By analyzing the wrapping time for a viral particle, the authors identified a threshold particle radius $(\sim 12 \mathrm{~nm}$ for cylindrical and $\sim 24 \mathrm{~nm}$ for spherical particles) and a threshold receptor density, below which clathrinindependent endocytosis would not occur. By considering the competition between thermodynamic driving force and receptor diffusion kinetics, an optimal particle radius for endocytosis was derived $(\sim 15 \mathrm{~nm}$ for cylindrical and $\sim 30 \mathrm{~nm}$ for spherical particles). Interestingly, the predicted results agreed remarkably well with experiments from Aoyama and colleagues [73-75]. Recently, the effect of elastic deformation of particles on cellular uptake was studied using a theoretical model [76]. The cellular internalization of particles was strongly dependent on the particle elasticity. Phase diagrams describing the transition between different wrapping phases were generated based on their analysis.
The continuum model developed by Sun and Wirtz [77] incorporated the energy contributions from the elastic deformations of the cell membrane and cytoskeleton, and the ligand-receptor interactions. From their analysis, the energy due to the deformation of cytoskeleton was much greater than the other energy contributions and this led to the conclusion that cytoskeleton deformation is the dominant determinant of endocytosis. However, as pointed out recently by Li et al. [78], the analysis in reference [77] underestimated the contribution from membrane deformation, and virus internalization should be dictated by the membrane deformation once the viral particle is beyond a critical size, which is dependent on the Young's modulus of cytoskeleton. Recent work by Decuzzi and Ferrari has considered the effects from the non-specific interactions [79] and particle nonsphericity [80] on receptor-mediated endocytosis (RME) for cylindrical particles. It was shown from their analysis that the contribution from non-specific interactions could be as important as those from specific ligand-receptor interactions; the particle shape and size also played critical roles in RME.

Recently, Zhang and coworkers [81] presented a theoretical analysis of the particle size effect on the number of nanoparticles (or virions) that can be internalized into the cell. Interestingly the optimal particle size for maximal cellular internalization predicted in their model agreed well with the predictions in reference [72]. Later the effects of particle size and ligand density on RME were discussed $[82,83]$. By analyzing the free energy functional, it was found that there existed a minimal particle size at a given ligand density and a minimal ligand density at a given particle size, below which endocytosis is not possible. The internalization rate interrelatedly depends on the particle size and ligand density, and an optimal condition (25-30 $\mathrm{nm}$ in particle radius and several tens of coated ligands) exists at which the endocytic rate is maximal. This finding is consistent with the structure of Semliki Forest virus, which has a radius of $\sim 35 \mathrm{~nm}$ and is covered with 80 glycoproteins.

\section{Stochastic mesoscale models}

A number of stochastic mesoscale models have been developed to study virus binding to host cell surfaces. In these models, the viral particles are treated as rigid spheres whose surfaces are decorated with ligand proteins. The viral particles bind to the cell surface through interactions between ligands and receptors on the cell surface. Both ligand and receptor proteins are coarsegrained, and the interaction potentials are determined from independent biophysical experiments. By accounting for the total force acting on the viral particles, the trajectories of viral particles can be calculated by solving the equations of motion. 
English and Hammer [84] implemented Brownian Adhesive Dynamics (BRAD) to simulate the receptormediated binding of viruses. In their model, the parameters were chosen to mimic the interactions between HIV particles and host cell surfaces. The cell membrane was treated as a rigid surface, and the kinetic rates of gp120CD4 binding were used as the ligand-receptor interactions. The binding of HIV particles to CD4-expressing cells were simulated using BRAD, and the results were compared with results predicted by an equivalent site hypothesis. Dramatic differences in the transition rates were found. In later contributions, CD4 receptor proteins were allowed to diffuse freely in the plane of the membrane [85], and the simple bead-spring model was developed to coarsely approximate the structure of glycoprotein gp120 [86]. Then the roles of cellular receptor diffusion and gp120 trimerization on HIV binding were investigated. The important finding was that it took seconds for gp120 to cluster CD4 in the contact zone, and this might partially explain the delay in viral entry. Using similar modeling strategies, Dobrowsky et al. [87] developed a stochastic model to investigate the spatial and temporal organization of cellular receptor CD4 and co-receptor CCR5 at the plasma membrane during HIV adhesion. In their model, the deformation of plasma membrane was evaluated by displacement of discrete points in Cartesian coordinates. CD4 and CCR5 were allowed to move on the membrane, and they were able to bind to gp120 on the HIV surface. The kinetic and micromechanical parameters for gp120-CD4 binding were obtained from direct experimental measurements [70]. A well-organized, ring-like, nanoscale structure beneath the virion, which the authors named the viral junction, was observed as a result of binding of gp120 to CD4 and CCR5. The local plasma membrane deformed in response to the formation of the viral junction. Similar structures were also reported in a tomographical image of HIV-1 in contact with T cells [53]. The authors speculated that the formation of the nanoscale viral junction might well correlate with the complex signaling events during viral entry, such as the assembly of a local actin network and the disassembly of actin filaments.

In drug delivery of functionalized nanocarriers (NCs) to endothelial cell (EC) surfaces, similar to RME of viral particles, NCs first bind to cell surface via ligandreceptor interaction and then enter the cell through RME. Recently, Liu et al. [88] developed a mesoscale binding model to study the drug delivery to EC surfaces using NCs. Briefly, in their model both the ligands on the NC surface (anti-ICAM1) and receptors on the EC surface (ICAM1) are coarse-grained as cylinders. The ICAM1s are allowed to diffuse on the EC surface, and the interactions and physical/mechanical parameters are determined from independent experiments. Using this model the authors developed a methodology to quantify the binding free energy or potential of mean force (PMF) between the NC and EC surfaces. Then the binding affinities were calculated based on the PMF profiles. The authors implemented this model to study the effect of anti-ICAM1 surface coverage of NC on binding and revealed a threshold value, below which the $\mathrm{NC}$ binding affinities decrease drastically and drop lower than that of a single anti-ICAM1 molecule to ICAM1. Interestingly, this prediction agrees remarkably well with experimental results of in vivo targeting of anti-ICAM1 coated NCs to pulmonary endothelium in mice. By analyzing the simulation results, it was revealed that the dominant effect of changing antibody surface coverage around the threshold is through a change in multivalent interactions. Moreover, the model results of NC rupture force distribution agree well with corresponding AFM experiments. The model was further extended to investigate effects of particle size, shear flow and resistance due to the existence of glycocalyx $[89,90]$. Intriguingly, all the model predictions agreed with the corresponding experiments. The mesoscale model developed in the context of drug delivery can be readily applied to study the binding of viral particles. A significant drawback in the above models is that the host cell membrane is either treated as a rigid surface or as a surface with small deformations. This restricts the discussions to the early adhesion of viral particles. A more flexible membrane model that can accommodate extreme deformations has been discussed in references [91,92], and is needed for these mesoscale models to analyze viral endocytic entry (see Figure 5 for illustration).

\section{Discrete models}

Full detailed molecular dynamics (MD) simulations are able to provide three-dimensional real-time information of the system with the finest atomistic level resolution. In principle, this can resolve all the structural and dynamic details. However, MD simulations are time consuming and are restricted to exploring systems with small spatial and temporal scales. For example, it will be difficult to simulate a lipid bilayer system consisting of more than hundreds of hydrated lipids for micron seconds using full detailed MD under current computational resources. Considering the spatial and temporal scales involved in viral endocytic entry, it is impractical to simulate using full detailed MD. Recently, a number of coarse-grained MD $[93,94]$ and dissipative particle dynamics (DPD) [95-98] simulations have been performed to explore the process of RME of nanoparticles (NPs). In such models, the lipid, ligand and receptor molecules are represented by a number of beads connected to each other. Each bead approximates the effect of many molecular atoms. The force on each bead and therefore the 


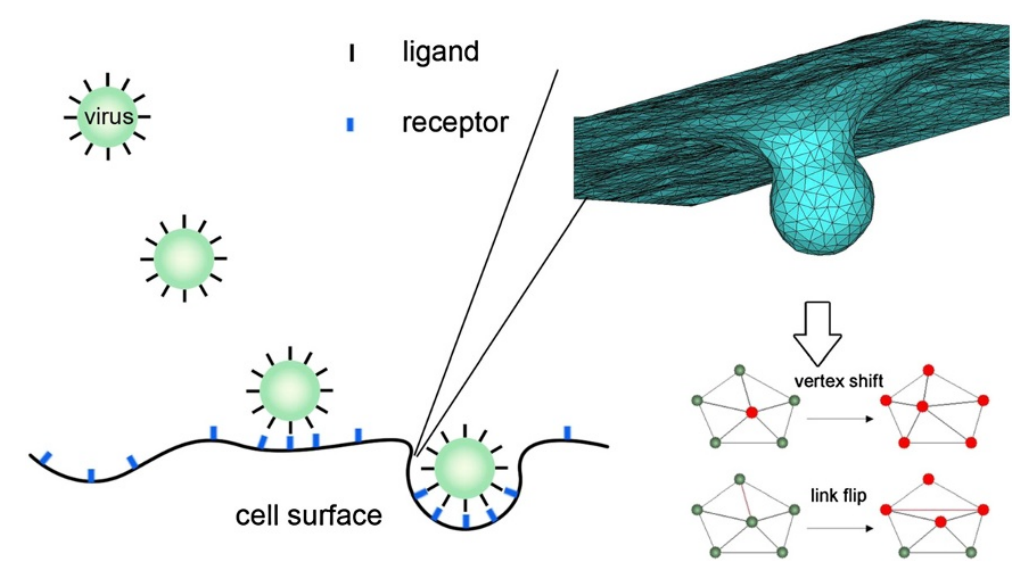

Figure $\mathbf{5}$ Schematic of the mesoscale model for virus endocytic entry. The virus is modeled as a sphere decorated with ligands. The cell surface is modeled as a plasma membrane with diffusive receptors. The membrane surface is discretized by a curvilinear triangulate system.

trajectory can be calculated through interaction potentials among different beads. In DPD, three types of forces, namely conservative, dissipative and random forces, are considered. The RME of NPs can be modeled by adjusting the interaction parameters. Through such coarse-graining techniques, the simulations can be extended to much larger spatial and temporal scales while retaining a certain degree of discrete information.

Yue and Zhang [95] presented a study on the receptor-mediated membrane responses to a ligandcoated NP using DPD simulations. Four types of membrane responses were observed in simulations: membrane rupture, NP adhesion, NP penetration and RME. The effects of NP size, membrane tension and ligand density on membrane response were discussed and phase diagrams were generated based on discussions. The effects of particle shape anisotropy on RME were studied in a later contribution [96]. Most recently the authors also investigated the pathways of the interaction between elastic vesicles and lipid membranes [98]. Using similar DPD simulations, Ding and Ma [97] have discussed the RME of NPs focusing on the effect of the coating ligand properties. Both the biochemical property (ligand-receptor interaction strength) and biophysical properties (length, rigidity and density) of the ligands are studied. Both biochemical and biophysical properties actively impact the efficiency of NP engulfment. Vacha et al. [93] have investigated the effects of size and shape of NPs on RME using coarse-grained MD simulations. Larger spherical particles entered the cell more readily than smaller ones due to a more favorable compromise between bending rigidity and surface adhesive energy. In addition, the spherocylindrical particles could be internalized more efficiently than spherical ones. Shi et al. [94] employed coarse-grained MD simulations to study the cell entry of carbon nanotubes. However, due to the computational cost, the sizes of the NPs (or vesicles) considered in these simulations are relatively small ( 10 $\mathrm{nm}$ in diameter).

\section{Conclusions}

Most viruses exploit endocytic pathways to enter cells to initiate infection. Thus a systematic and mechanistic understanding of the virus endocytic entry process is critically important for the development of targeted and specific inhibitors of virus entry and infection [99]. The events involved in the process are dynamic and span multiple spatial and temporal scales. Information about the process is accumulating with the advent of new and powerful experimental technologies as we have discussed in section 2. However, the experimental complexity of a single technique usually restricts observations to a specific spatial and temporal scale and therefore can only describe part of the whole story. Optical and electron microscopy are able to directly provide information on a cellular level but neglect molecular level events. Singlemolecule force spectroscopy is able to probe interactions at the molecular level but overlooks the effects of the microenvironment. The question of how to interpret and integrate these seemingly scale-specific data and provide a systematic view of the whole process is a significant challenge.

From a modeling point of view, similar situations are present. Ideally we hope to model the whole viral endocytic entry process with resolution at the molecular scale. Theoretical continuum models (macroscale models) have the advantage of efficiency and are able to resolve cellular level events, but the molecular scale details cannot be resolved. On the other hand, discrete atomistic models (microscale models) are able to provide all the molecular details but are computationally prohibitive to simulating a realistic system. As a result, recently much effort has been devoted to the development of multiscale models. In such models, a microscale 
model is integrated into a macroscale model so that the expensive microscale calculations only take place in necessary places. By proper design of the communications across different models (scales), a multiscale model has the advantages of both the efficiency of the macroscale models and the accuracy of the microscale models. The biggest challenge in a multiscale model is how to handle the interfaces between different models where information should be mutually exchanged. Some methodologies have been developed, though, in other disciplines [100-104].

Finally, the complexity and multiscale nature of the viral endocytic entry process requires investigation at a systems biology level. This demands interdisciplinary knowledge and expertise in cellular, structural and molecular biology, as well as the collaborative and complementary work between experimental and theoretical/modeling research. Here we would like to emphasize the important role of collaboration between experimentalists and theorists. Theoretical and numerical models have the power of mechanistically interpreting data uncovered in experiments, and making predictions to guide further experimental design. However, all models must eventually be faithful to experiments and must be rigorously validated through experimentation before making predictions. The model parameters must be evaluated from the corresponding experiments. Furthermore, a model should be versatile and adaptive to modifications to incorporate new important factors discovered by experimentation. This process involves iterative validation and verification between modeling and experiments. With the emerging novel and more quantitative experimental technologies, as well as the new modeling methodologies, especially the multiscale modeling methods, a definitive and mechanistic understanding of viral endocytic entry is taking place.

\section{Competing interests}

The authors declare they have no competing interests.

\section{Authors' contributions}

$E B, A V N$, and JL participated in the writing of the manuscript. All authors have read and approved the final manuscript.

\section{Acknowledgements \\ Work in the authors' laboratories was supported by National Science Foundation (NSF) grant CBET-1250107 (JL) and Public Health Service grant Al-096103 from the National Institute of Allergy and Infectious Diseases (AVN).}

\section{Author details}

${ }^{1}$ School of Mechanical and Materials Engineering, Washington State University, Pullman, WA 99164, USA. ²Department of Veterinary Microbiology and Pathology, Washington State University, Pullman, WA 99164, USA. ${ }^{3}$ Paul G. Allen School for Global Animal Health, Washington State University, Pullman, WA 99164, USA.

Received: 24 April 2013 Accepted: 24 May 2013

Published: 5 June 2013

\section{References}

1. Enquist LW: Virology in the 21st century. J Virol 2009, 83:5296-5308.

2. Fauci AS: Emerging and re-emerging infectious diseases: influenza as a prototype of the host-pathogen balancing act. Cell 2006, 124:665-670.

3. Morens DM, Folkers GK, Fauci AS: Emerging infections: a perpetual challenge. Lancet Infect Dis 2008, 8:710-719.

4. Smith AE, Helenius A: How viruses enter animal cells. Science 2004, 304:237-242.

5. Mercer J, Schelhaas M, Helenius A: Virus entry by Endocytosis. Annu Rev Biochem 2010, 79:803-833.

6. Sieczkarski SB, Whittaker GR: Dissecting virus entry via endocytosis. J Gen Virol 2002, 83:1535-1545.

7. Pelkmans $L$, Helenius $A$ : Insider information: what viruses tell us about endocytosis. Curr Opin Cell Biol 2003, 15:414-422.

8. Marsh M, Helenius A: Virus entry: open sesame. Cell 2006, 124:729-740.

9. Doherty GJ, McMahon HT: Mechanisms of endocytosis. Annu Rev Biochem 2009, 78:857-902.

10. Ramanan V, Agrawal NJ, Liu J, Engles S, Toy R, Radhakrishnan R: Systems biology and physical biology of clathrin-mediated endocytosis. Integr Biol 2011, 3:803-815

11. Canton I, Battaglia G: Endocytosis at the nanoscale. Chem Soc Rev 2012, 41:2718-2739.

12. Miyauchi K, Kim Y, Latinovic O, Morozov V, Melikyan GB: HIV enters cells via endocytosis and dynamin-dependent fusion with endosomes. Cell 2009, 137:433-444.

13. Mercer J, Helenius A: Virus entry by macropinocytosis. Nat Cell Bio/ 2009, 11:510-520.

14. Nicola AV, Hou J, Major EO, Straus SE: Herpes simplex virus type 1 enters human epidermal keratinocytes, but not neurons, via a $\mathrm{pH}$-dependent endocytic pathway. J Virol 2005, 79:7609-7616.

15. Amstutz B, Gastaldelli M, Kalin S, Imelli N, Boucke K, Wandeler E, Mercer J, Hemmi S, Greber UF: Subversion of CtBP1-controlled macropinocytosis by human adenovirus serotype 3. EMBO J 2008, 27:956-969.

16. Mercer J, Helenius A: Vaccinia virus uses macropinocytosis and apoptotic mimicry to enter host cells. Science 2008, 320:531-535.

17. Stang E, Kartenbeck J, Parton RG: Major histocompatibility complex class I molecules mediate association of SV40 with caveolae. Mol Biol Cell 1997, 8:47-57.

18. Richterova Z, Liebl D, Horak M, Palkova Z, Stokrova J, Hozak P, Korb J, Forstova J: Caveolae are involved in the trafficking of mouse polyomavirus virions and artificial VP1 pseudocapsids toward cell nuclei. J Virol 2001, 75:10880-10891.

19. Pelkmans L, Helenius A: Endocytosis via caveolae. Traffic 2002, 3:311-320.

20. Sieczkarski SB, Whittaker GR: Influenza virus can enter and infect cells in the absence of clathrin-mediated endocytosis. J Virol 2002, 76:10455-10464.

21. Lakadamyali M, Rust MJ, Zhuang XW: Endocytosis of influenza viruses. Microbes Infect 2004, 6:929-936.

22. Rust MJ, Lakadamyali M, Zhang F, Zhuang XW: Assembly of endocytic machinery around individual influenza viruses during viral entry. Nat Struct Mol Biol 2004, 11:567-573.

23. Pelkmans L, Fava E, Grabner H, Hannus M, Habermann B, Krausz E, Zerial M: Genome-wide analysis of human kinases in clathrin- and caveolae/raftmediated endocytosis. Nature 2005, 436:78-86.

24. Damm E-M, Pelkmans L: Systems biology of virus entry in mammalian cells. Cell Microbiol 2006, 8:1219-1227.

25. Greber UF: Signalling in viral entry. Cell Mol Life Sci 2002, 59:608-626

26. Gruenberg J: Viruses and endosome membrane dynamics. Curr Opin Cell Biol 2009, 21:582-588.

27. Mayor S, Pagano RE: Pathways of clathrin-independent endocytosis. Nat Rev Mol Cell Biol 2007, 8:603-612.

28. Dales S: An electron microscope study of the early association between two mammalian viruses and their hosts. J Cell Biol 1962, 13:303-322.

29. Nicola AV, McEvoy AM, Straus SE: Roles for endocytosis and Low pH in herpes simplex virus entry into HeLa and chinese hamster ovary cells. J Virol 2003, 77:5324-5332.

30. Brandenburg $B$, Zhuang $X$ : Virus trafficking - learning from single-virus tracking. Nat Rev Microbiol 2007, 5:197-208.

31. Pelkmans L, Kartenbeck J, Helenius A: Caveolar endocytosis of simian virus 40 reveals a new two-step vesicular-transport pathway to the ER. Nat Cell Biol 2001, 3:473-483. 
32. Chen $C$, Zhuang $X$ : Epsin 1 is a cargo-specific adaptor for the clathrin-mediated endocytosis of the influenza virus. Proc Natl Acad Sci USA 2008, 105:11790-11795.

33. van der Schaar HM, Rust MJ, Chen C, van der Ende-Metselaar H, Wilschut J, Zhuang $X$, Smit JM: Dissecting the cell entry pathway of dengue virus by single-particle tracking in living cells. PLoS Pathog 2008, 4:e1000244.

34. Ewers $H$, Smith AE, Sbalzarini IF, Lilie H, Koumoutsakos P, Helenius A: Single-particle tracking of murine polyoma virus-like particles on live cells and artificial membranes. Proc Natl Acad Sci USA 2005, 102:15110-15115.

35. Schelhaas M, Ewers H, Rajamaki M-L, Day PM, Schiller JT, Helenius A: Human papillomavirus type 16 entry: retrograde cell surface transport along actin-rich protrusions. PLoS Pathog 2008, 4:e1000148.

36. Brandenburg B, Lee LY, Lakadamyali M, Rust MJ, Zhuang X, Hogle JM: Imaging poliovirus entry in live cells. PLOS Biol 2007, 5:1543-1555.

37. Vaughan JC, Brandenburg B, Hogle JM, Zhuang X: Rapid actin-dependent viral motility in live cells. Biophys J 2009, 97:1647-1656.

38. Huang B, Bates M, Zhuang X: Super-Resolution Fluorescence Microscopy In Annu Rev Biochem, Volume 78; 2009:993-1016. Annual Review of Biochemistry.

39. Rust MJ, Bates M, Zhuang X: Sub-diffraction-limit imaging by stochastic optical reconstruction microscopy (STORM). Nat Methods 2006, 3:793-795.

40. Betzig E, Patterson GH, Sougrat R, Lindwasser OW, Olenych S, Bonifacino JS, Davidson MW, Lippincott-Schwartz J, Hess HF: Imaging intracellular fluorescent proteins at nanometer resolution. Science 2006, 313:1642-1645.

41. Gustafsson MGL: Nonlinear structured-illumination microscopy: wide-field fluorescence imaging with theoretically unlimited resolution. Proc Natl Acad Sci USA 2005, 102:13081-13086.

42. Pereira CF, Rossy J, Owen DM, Mak J, Gaus K: HIV taken by STORM: superresolution fluorescence microscopy of a viral infection. Virol J 2012, 9:84

43. Rossman JS, Leser GP, Lamb RA: Filamentous influenza virus enters cells via macropinocytosis. J Virol 2012, 86:10950-10960.

44. Subramaniam S, Bartesaghi A, Liu J, Bennett AE, Sougrat R: Electron tomography of viruses. Curr Opin Struct Biol 2007, 17:596-602.

45. Grunewald K, Desai P, Winkler DC, Heymann JB, Belnap DM, Baumeister W, Steven AC: Three-dimensional structure of herpes simplex virus from cryo-electron tomography. Science 2003, 302:1396-1398.

46. Forster F, Medalia O, Zauberman N, Baumeister W, Fass D: Retrovirus envelope protein complex structure in situ studied by cryo-electron tomography. Proc Natl Acad Sci USA 2005, 102:4729-4734.

47. Cyrklaff M, Risco C, Fernandez JJ, Jimenez MV, Esteban M, Baumeister W, Carrascosa JL: Cryo-electron tomography of vaccinia virus. Proc Natl Acad Sci USA 2005, 102:2772-2777.

48. Zanetti G, Briggs JAG, Gruenewald K, Sattentau QJ, Fuller SD: Cryo-electron tomographic structure of an immunodeficiency virus envelope complex in situ. PLOS Pathog 2006, 2:e83.

49. Zhu P, Liu J, Bess J, Chertova E, Lifson JD, Grise H, Ofek GA, Taylor KA, Roux $\mathrm{KH}$ : Distribution and three-dimensional structure of AIDS virus envelope spikes. Nature 2006, 441:847-852.

50. Harris A, Cardone G, Winkler DC, Heymann JB, Brecher M, White JM, Steven AC: Influenza virus pleiomorphy characterized by cryoelectron tomography. Proc Natl Acad Sci USA 2006, 103:19123-19127.

51. Bharat TAM, Noda T, Riches JD, Kraehling V, Kolesnikova L, Becker S, Kawaoka Y, Briggs JAG: Structural dissection of Ebola virus and its assembly determinants using cryo-electron tomography. Proc Natl Acad Sci USA 2012, 109:4275-4280.

52. Iwasaki K, Omura T: Electron tomography of the supramolecular structure of virus-infected cells. Curr Opin Struct Biol 2010, 20:632-639.

53. Sougrat R, Bartesaghi A, Lifson JD, Bennett AE, Bess JW, Zabransky DJ, Subramaniam S: Electron tomography of the contact between T cells and SIV/HIV-1: implications for viral entry. PLoS Pathog 2007, 3:571-581.

54. Maurer UE, Sodeik B, Gruenewald K: Native 3D intermediates of membrane fusion in herpes simplex virus 1 entry. Proc Natl Acad Sci USA 2008, 105:10559-10564.

55. Peng L, Ryazantsev S, Sun R, Zhou ZH: Three-dimensional visualization of gammaherpesvirus life cycle in host cells by electron tomography. Structure 2010, 18:47-58.

56. Cyrklaff M, Linaroudis A, Boicu M, Chlanda P, Baumeister W, Griffiths G, Krijnse-Locker J: Whole cell cryo-electron tomography reveals distinct disassembly intermediates of vaccinia virus. PLoS One 2007, 2:e420.
57. Welsch S, Kolesnikova L, Kraehling V, Riches JD, Becker S, Briggs JAG: Electron tomography reveals the steps in filovirus budding. PLOS Pathog 2010, 6:e1000875.

58. Grove J, Marsh M: The cell biology of receptor-mediated virus entry. J Cell Biol 2011, 195:1071-1082

59. Willis SH, Rux AH, Peng C, Whitbeck JC, Nicola AV, Lou H, Hou WF, Salvador $\mathrm{L}$, Eisenberg RJ, Cohen $\mathrm{GH}$ : Examination of the kinetics of herpes simplex virus glycoprotein D binding to the herpesvirus entry mediator, using surface plasmon resonance. J Virol 1998, 72:5937-5947.

60. Milne RSB, Hanna SL, Rux AH, Willis SH, Cohen GH, Eisenberg RJ: Function of herpes simplex virus type $1 \mathrm{gD}$ mutants with different receptorbinding affinities in virus entry and fusion. J Virol 2003, 77:8962-8972.

61. Alessandrini A, Facci P: AFM: a versatile tool in biophysics. Meas SCi Technol 2005, 16:R65-R92.

62. Alessandrini A, Facci P: Unraveling lipid/protein interaction in model lipid bilayers by atomic force microscopy. J Mol Recognit 2011, 24:387-396.

63. Ovalle-Garcia E, Torres-Heredia JJ, Antillon A, Ortega-Blake I: Simultaneous determination of the elastic properties of the lipid bilayer by atomic force microscopy: bending, tension, and adhesion. J Phys Chem B 2011 , 115:4826-4833.

64. Cross SE, Jin Y-S, Rao J, Gimzewski JK: Nanomechanical analysis of cells from cancer patients. Nat Nanotechnol 2007, 2:780-783.

65. Iyer S, Gaikwad RM, Subba-Rao V, Woodworth CD, Sokolov I: Atomic force microscopy detects differences in the surface brush of normal and cancerous cells. Nat Nanotechnol 2009, 4:389-393.

66. Wirtz D, Konstantopoulos K, Searson PC: The physics of cancer: the role of physical interactions and mechanical forces in metastasis. Nat Rev Cancer 2011, 11:512-522.

67. McDougal JS, Kennedy MS, Sligh JM, Cort SP, Mawle A, Nicholson JKA: Binding of HTLV-III/LAV to T4\$^\{+\}\$T cells by a complex of the $110 \mathrm{~K}$ viral protein and the T4 molecule. Science 1986, 231:382-385.

68. Lasky LA, Nakamura G, Smith DH, Fennie C, Shimasaki C, Patzer E, Berman P, Gregory T, Capon DJ: Delineation of a region of the human immunodeficiency virus type $1 \mathrm{gp} 120$ glycoprotein critical for interaction with the CD4 receptor. Cell 1987, 50:975-985.

69. Chang MI, Panorchan P, Dobrowsky TM, Tseng Y, Wirtz D: Single-molecule analysis of human immunodeficiency virus type $1 \mathrm{gp} 120$-receptor interactions in living cells. J Virol 2005, 79:14748-14755.

70. Dobrowsky TM, Zhou Y, Sun SX, Siliciano RF, Wirtz D: Monitoring early fusion dynamics of human immunodeficiency virus type 1 at single-molecule resolution. J Virol 2008, 82:7022-7033.

71. van Effenterre D, Roux D: Adhesion of colloids on a cell surface in competition for mobile receptors. Europhys Lett 2003, 64:543-549.

72. Gao HJ, Shi WD, Freund LB: Mechanics of receptor-mediated endocytosis. Proc Natl Acad Sci USA 2005, 102:9469-9474

73. Aoyama Y, Kanamori T, Nakai T, Sasaki T, Horiuchi S, Sando S, Niidome T: Artificial viruses and their application to gene delivery. Size-controlled gene coating with glycocluster nanoparticles. J Am Chem Soc 2003, 125:3455-3457.

74. Nakai T, Kanamori T, Sando S, Aoyama Y: Remarkably size-regulated cell invasion by artificial viruses. Saccharide-dependent self-aggregation of glycoviruses and its consequences in glycoviral gene delivery. J Am Chem Soc 2003, 125:8465-8475.

75. Osaki F, Kanamori T, Sando S, Sera T, Aoyama Y: A quantum dot conjugated sugar ball and its cellular uptake on the size effects of endocytosis in the subviral region. J Am Chem Soc 2004, 126:6520-6521.

76. Yi X, Shi X, Gao H: Cellular uptake of elastic nanoparticles. Phys Rev Lett 2011, 107:098101.

77. Sun SX, Wirtz D: Mechanics of enveloped virus entry into host cells. Biophys J 2006, 90:L10-L12.

78. Li L, Liu $X$, Zhou Y, Wang J: On resistance to virus entry into host cells. Biophys J 2012, 102:2230-2233.

79. Decuzzi P, Ferrari M: The role of specific and non-specific interactions in receptor-mediated endocytosis of nanoparticles. Biomaterials 2007, 28:2915-2922

80. Decuzzi P. Ferrari M: The receptor-mediated endocytosis of nonspherical particles. Biophys J 2008, 94:3790-3797.

81. Zhang S, Li J, Lykotrafitis G, Bao G, Suresh S: Size-dependent endocytosis of nanoparticles. Adv Mater 2009, 21:419-424

82. Yuan $\mathrm{H}$, Huang $\mathrm{C}$, Zhang S: Virus-inspired design principles of nanoparticle-based bioagents. PLoS One 2010, 5:e13495. 
83. Yuan $\mathrm{H}$, Zhang $\mathrm{S}$ : Effects of particle size and ligand density on the kinetics of receptor-mediated endocytosis of nanoparticles. Appl Phys Lett 2010, 96:033704

84. English TJ, Hammer DA: Brownian adhesive dynamics (BRAD) for simulating the receptor-mediated binding of viruses. Biophys J 2004, 86:3359-3372.

85. English TJ, Hammer DA: The effect of cellular receptor diffusion on receptor-mediated viral binding using brownian adhesive dynamics (BRAD) simulations. Biophys J 2005, 88:1666-1675.

86. Trister AD, Hammer DA: Role of gp120 trimerization on HIV binding elucidated with brownian adhesive dynamics. Biophys J 2008, 95:40-53.

87. Dobrowsky TM, Daniels BR, Siliciano RF, Sun SX, Wirtz D: Organization of cellular receptors into a nanoscale junction during HIV-1 adhesion. PLoS Comput Biol 2010, 6:e1000855.

88. Liu J, Weller GER, Zern B, Ayyaswamy PS, Eckmann DM, Muzykantov VR, Radhakrishnan R: Computational model for nanocarrier binding to endothelium validated using in vivo, in vitro, and atomic force microscopy experiments. Proc Natl Acad Sci USA 2010, 107:16530-16535.

89. Liu J, Agrawal NJ, Calderon A, Ayyaswamy PS, Eckmann DM, Radhakrishnan R: Multivalent binding of nanocarrier to endothelial cells under shear flow. Biophys $J$ 2011, 101:319-326.

90. Liu J, Bradley R, Eckmann DM, Ayyaswamy PS, Radhakrishnan R: Multiscale modeling of functionalized nanocarriers in targeted drug delivery. Curr Nanosci 2011, 7:727-735.

91. Ramakrishnan N, Kumar PBS, Ipsen JH: Monte Carlo simulations of fluid vesicles with in-plane orientational ordering. Phys Rev E 2010, 81:041922.

92. Liu J, Tourdot R, Ramanan V, Agrawal NJ, Radhakrishanan R: Mesoscale simulations of curvature-inducing protein partitioning on lipid bilayer membranes in the presence of mean curvature fields. Mol Phys 2012, 110:1127-1137.

93. Vacha R, Martinez-Veracoechea FJ, Frenkel D: Receptor-mediated endocytosis of nanoparticles of various shapes. Nano Lett 2011, 11:5391-5395.

94. Shi X, von dem Bussche A, Hurt RH, Kane AB, Gao H: Cell entry of one-dimensional nanomaterials occurs by tip recognition and rotation. Nat Nanotechnol 2011, 6:714-719.

95. Yue T, Zhang X: Molecular understanding of receptor-mediated membrane responses to ligand-coated nanoparticles. Soft Matter 2011 7:9104-9112.

96. Li Y, Yue T, Yang K, Zhang X: Molecular modeling of the relationship between nanoparticle shape anisotropy and endocytosis kinetics. Biomaterials 2012, 33:4965-4973.

97. Ding $\mathrm{H}-\mathrm{m}, \mathrm{Ma} \mathrm{Y}-\mathrm{q}$ : Role of physicochemical properties of coating ligands in receptor-mediated endocytosis of nanoparticles. Biomaterials 2012, 33:5798-5802

98. Yue T, Zhang X: Molecular modeling of the pathways of vesiclemembrane interaction. Soft Matter 2013, 9:559-569.

99. Teissier E, Penin F, Pecheur E-l: Targeting cell entry of enveloped viruses as an antiviral strategy. Molecules 2011, 16:221-250.

100. O'Connell ST, Thompson PA: Molecular dynamics-continuum hybrid computations: a tool for studying complex fluid flows. Phys Rev E 1995, 52:R5792-R5795.

101. Hadjiconstaninou NG, Patera AT: Heterogeneous atomistic-continuum representations for dense fluid systems. Int J Mod Phys C 1997, 8:967-976.

102. Flekkoy EG, Wagner $G$, Feder J: Hybrid model for combined particle and continuum dynamics. Europhys Lett 2000, 52:271-276.

103. E W, Engquist B, Li X, Ren W, Vanden-Eijnden E: Heterogeneous multiscale methods: A review. Commun Comput Phys 2007, 2:367-450.

104. Liu J, Chen SY, Nie XB, Robbins MO: A continuum-atomistic simulation of heat transfer in micro- and nano-flows. J Comput Phys 2007, 227:279-291.

doi:10.1186/1743-422X-10-177

Cite this article as: Barrow et al:: Multiscale perspectives of virus entry

via endocytosis. Virology Journal 2013 10:177.

\section{Submit your next manuscript to BioMed Central and take full advantage of:}

- Convenient online submission

- Thorough peer review

- No space constraints or color figure charges

- Immediate publication on acceptance

- Inclusion in PubMed, CAS, Scopus and Google Scholar

- Research which is freely available for redistribution

Submit your manuscript at www.biomedcentral.com/submit 